\title{
ABSTRACTS DER MASTERTHESEN DES INTERUNIVERSITÄREN UNIVERSITÄTSLEHRGANGES LIBRARY AND INFORMATION STU- DIES 2017
}

\section{übermittelt von Gabriele Pum}

Im Rahmen des Interuniversitären Universitätslehrganges Library and Information Studies an der Universität Wien in Kooperation mit der Österreichischen Nationalbibliothek leisten die AbsolventInnen mit ihren Master Thesen einen wichtigen Beitrag zum Forschungsoutput im Fachbereich Library and Information Studies in Österreich im universitären Rahmen. Im Jahr 2017 wurden in Wien folgende Master Thesen abgeschlossen:

\section{Stefanie Astrid Andergassen BA}

\section{Das Publikationsverhalten der Rechtswissenschaften am Beispiel der Wirt- schaftsuniversität Wien}

In der vorliegenden Master Thesis werden die Besonderheiten des Publikationsverhaltens der Rechtswissenschaften erörtert und anhand einer Analyse der Publikationsdaten der Wirtschaftsuniversität (WU) Wien belegt. Diese Besonderheiten des rechtswissenschaftlichen Publizierens werden in den aktuellen Untersuchungen zum Publikationsverhalten vernachlässigt oder mit denen der Sozial- und Geisteswissenschaften generalisiert dargestellt.

Zunächst soll eine Definition für den Begriff Publikationsverhalten gefunden werden. Einhergehend mit der Definition ist die Festlegung von Kriterien, welche das Publikationsverhalten kennzeichnen. Dazu zählen Kriterien wie Publikationsform, Publikationsort, Autorenschaft oder auch Publikationssprache und markieren damit welche, wie, für wen und wo Inhalte veröffentlicht werden. Im weiteren Verlauf der Master Thesis werden diese anfänglich festgelegten Publikationskriterien herangezogen um die Besonderheiten des Publikationsverhaltens der Rechtswissenschaften darzulegen.

Die methodische Vorgehensweise besteht primär aus einer umfassenden Darstellung der bereits publizierten Literatur zum Thema Publikationsverhalten in den Rechtswissenschaften und einer detaillierten Analyse der Publikationsdaten der WU Wien aus dem Jahr 2015. Die Auswertung der Publikationsdaten zeigt unter anderem eine Dominanz von Publikationen in 
der Landessprache Deutsch, überproportional viele Einzelautorenschaften und im Kontrast zu den Fächern Science, Technology and Medicine (STM) vermehrt Veröffentlichungen von monographischen Publikationsformen.

Es wird schlussfolgernd dargestellt, dass aufgrund dieser speziellen rechtswissenschaftlichen Publikationsverhaltensweisen gängige bibliometrische Analysen ungeeignet sind. Denn Analysen basierend auf den Zitationsdatenbanken des Web of Science sind auf das Publikationsverhalten der STM-Fächer zugeschnitten und können deshalb zu Fehlern und Verzerrungen bei der rechtswissenschaftlichen Leistungsevaluation führen.

\section{Mag. ${ }^{a}$ Elisabeth Bacher}

\section{Benutzerforschung in öffentlichen Bibliotheken am Beispiel der Stadtbücherei Innsbruck}

Öffentliche Bibliotheken verstehen sich als Bildungs-, Kultur-, Informations- und Integrationszentren. Ihre Hauptaufgabe ist die Schaffung eines niederschwelligen Zugangs zu Bildung und Information. Durch die wachsenden Ansprüche unserer Gesellschaft sehen sich Bibliotheken jedoch mit Herausforderungen konfrontiert: Neue Medien(nutzung) und gesellschaftspolitischer Wandel machen ein Umdenken notwendig.

Auch im Bereich der NutzerInnenorientierung erfährt der Bibliothekssektor einen Wandel. Es werden immer öfter Befragungen durchgeführt zur Serviceoptimierung durchgeführt. Ein solcher Erkenntnisprozess wird anhand einer Umfrage in der Stadtbücherei Innsbruck erläutert. Die Institution will für ihren Neubau wissen, wie die Einrichtung genutzt wird und welche Services beibehalten werden sollen oder einer Verbesserung bedürfen. Gleichzeitig sollen Trends aus den Bibliotheks-wissenschaften in die Neukonzeptionierung einfließen, um eine zeitgemäße Bibliotheksarbeit zu gewährleisten.

Die Umfrage ergab, dass das Angebot vermehrt auf Familien ausgerichtet werden muss, Leseförderung und Weiterbildungsangebote für Erwachsene und Migrantlnnen an Bedeutung gewinnen und dass der Bestand ausgebaut und erneuert werden muss. Aktualität und Verfügbarkeit bedürfen einer Verbesserung. Viele Projekte, Veranstaltungsreihen und Werbekanäle sind veraltet und müssen neu aufgestellt werden, um besser wahrgenommen zu werden. Die Bücherei wird zunehmend als Aufenthaltsort genützt, was eine räumliche Umgestaltung und Vergrößerung erfordert. Gute Beratung durch ausgebildetes Fachpersonal muss verstärkt und die Öffnungszeiten 
dem Berufsleben durch Abend- und Wochenend-öffnungszeiten angepasst werden. Konzepte und Strategien unterstützen die Umsetzung dieser Anforderungen, wie z. B. in Form von Kooperationen mit anderen Institutionen, eines Mitarbeiterlnnenausbaus mit vielfältigen Qualifikationen, welche Projekte, Veranstaltungen, Öffentlichkeitsarbeit und Bestand neu aufstellen sowie eine durchdachte räumliche Aufteilung für alle Bereiche und Technik, die modernsten Standards entspricht und neue Projekte ermöglicht.

\section{Lina Bittner B.A.}

\section{Das Bücherdorf - eine neue Form des Literaturtourismus. Eine Untersuchung unter Berücksichtigung wirtschaftlicher, kultureller und gesellschaftlicher As- pekte}

Diese Arbeit behandelt Literaturtourismus und wirtschaftliche, gesellschaftliche und kulturelle Aspekte, die mit diesem in Zusammenhang stehen. Zum einen wird untersucht ob Bücherdörfer Teil des Literaturtourismus sind, zum anderen besteht eine Aufgabe der Arbeit darin, Purgstall als erstes österreichisches Bücherdorf zu evaluieren. Literaturtourismus ist eine Form des Tourismus, die seit Jahrtausenden besteht, in den letzten Jahrzehnten jedoch einen starken Aufschwung erfahren hat. Literaturtourismus kann vielfältige Formen annehmen und die wirtschaftliche und kulturelle Entwicklung eines Orts entscheidend beeinflussen, während die Bewahrung der Authentizität von literaturtouristischen Sehenswürdigkeiten eine ständige Herausforderung darstellt. Die Entstehung von Bücherdörfern ist eine Entwicklung, die die Tourismusindustrie der letzten 50 Jahre entscheidend geprägt hat. Die Umwandlung eines Orts in ein Bücherdorf beeinflusst sowohl die wirtschaftliche und gesellschaftliche Entwicklung der Umgebung als auch kulturelle Aktivitäten im Umland.

Sowohl Literaturtourismus als auch Buchdorftourismus werden in Bezug auf Aspekte wie Anwohnerlnnen, Besucherlnnen, Wirtschaftlichkeit, Kulturvermittlung und Authentizität untersucht. Des Weiteren sollen Instrumente wie eine Umfrage, eine Markt- und eine SWOT-Analyse eine mögliche Richtung für die zukünftige Entwicklung des Bücherdorfs Purgstall vorgeben. Anhand dessen werden Erfolgsfaktoren bestimmt, die für den Fortbestand ähnlicher Projekte entscheidend sind. Abschließend werden die wichtigsten Aspekte des Literatur- und Bücherdorftourismus miteinander verglichen. Ziel dieser Arbeit ist die Bestätigung der These, dass der Besuch von Bücherdörfern eine neue Form des Literaturtourismus darstellt. 


\section{Der Umgang mit Requirements-Engineering an wissenschaftlichen Biblio- theken. State of the Art und Zukunftsperspektiven}

Die Arbeit beschäftigt sich mit dem Umgang mit technischen Anforderungen an wissenschaftlichen Bibliotheken. Während in der Wirtschaft Requirements-Engineering bereits seit langer Zeit eingesetzt wird um einen reibungslosen Ablauf von IT-Projekten zu garantieren, beschäftigen sich Bibliotheken bisher kaum mit diesem Thema, obwohl auch hier die Zusammenarbeit mit dem IT-Bereich immer wichtiger wird, z.B. durch die Bereitstellung von forschungsunterstützenden Services. Requirements-Engineers sind wie Vermittlerlnnen zwischen den Auftraggeberlnnen und den IT-KollegInnen zu sehen. Sie bereiten zunächst das Projekt vor, nehmen Kontakt mit allen Stakeholdern auf, ermitteln die Anforderungen, formulieren und prüfen sie und begleiten den gesamten Prozess bis zur Umsetzung. Ein Ziel der Arbeit war anhand ausgewählter Literatur einen Überblick über den professionelle Umgang mit Anforderungen zu geben, ein Jobprofil für Requirements-Engineers an Bibliotheken zu entwerfen und ein Stimmungsbild, wie derzeit an österreichischen wissenschaftlichen Institutionen mit Anforderungen umgegangen wird, zu zeigen. Dazu wurden VertreterInnen - BibliothekarInnen und TechnikerInnen - aus insgesamt 13 Institutionen befragt. Das Ergebnis bestätigte die These, dass Requirements-Engineering in Bibliotheken noch weitgehend unbekannt ist, bzw. nicht eingesetzt wird. Allerdings werden bereits zahlreiche Projekte zwischen verschiedenen Abteilungen von Bibliotheken und IT-Abteilungen, bzw. Firmen erfolgreich durchgeführt. Beklagt wurden sehr oft die mangelhafte Ausbildung des Personals im technischen Bereich und die Tatsache, dass für Projekte allgemein zu wenig Geld und Personal vorhanden wären. Würde man hier mehr investieren, könnten sich Bibliotheken auf lange Sicht Zeit und Kosten sparen. Eine kurze Übersicht, was in Zukunft beachtet werden sollte, sowie die Empfehlung Requirements-Engineering in den Lehrplan der Bibliotheksausbildung aufzunehmen runden die Arbeit ab.

Mag. Franz Halas

Büchereien und Bundespolitik. Das Bundesgesetz über die Förderung der Erwachsenenbildung und des Volksbüchereiwesens aus Bundesmitteln - Gesetz und Wirklichkeit 
Die Arbeit untersucht die Entwicklung und Rechtslage in Bezug auf die Förderpolitik des Bundes gegenüber öffentlichen Büchereien in Österreich. Ausgangspunkt der Untersuchung bildet das Bundesgesetz über die Förderung der Erwachsenenbildung und des Volksbüchereiwesens aus Bundesmitteln vom 21. März 1973. Es werden anhand von Quellen und Sekundärliteratur dessen historische Entwicklung und die Auswirkungen auf das Büchereiwesen dargestellt und analysiert. Thematisiert wird zudem die Verortung des Büchereiwesens innerhalb des Sektors Erwachsenenbildung und dessen Sonderstellung darin, die bereits durch die explizite Nennung des Volksbüchereiwesens im Gesetzestitel deutlich wird. Anhand der strukturellen Veränderungen aufgrund der letzten Gesetzesänderung von 2003 wird der Wandel der Förderpolitik dargestellt. Hierbei ist vor allem auf die Auflösung der Förderungsstellen des Bundes für Erwachsenenbildung und die daran angeschlossenen Büchereistellen zu verweisen, da diese einen Verzicht des Bundes auf ein direktes Steuerungsinstrument in diesem gesellschaftlichen Subsystem darstellte. Im Büchereiwesen führte dies zu einer Stärkung der Verbandswesens - vor allem der Büchereiverband Österreichs wurde nach der legistisch bedingten Umstrukturierung als zentrales Organisationselement in der Förderungsvergabe etabliert. Die Analyse der derzeitigen Förderstrukturen auf Bundesebene zeigt dann bestehende Stärken und Problemlagen. Abschließend werden die Ergebnisse zusammengefasst und eine Bewertung des Verhältnisses von Bundespolitik und Büchereiwesen vorgenommen.

\section{Mag. ${ }^{a}$ Manuela Leutgeb}

\section{Sondermaterialien in der Wienbibliothek im Rathaus - eine Analyse}

Die Arbeit widmet sich der Thematik Einblattdrucke, Flugblätter und Flugschriften und zeichnet exemplarisch die Erschließung und Digitalisierung ausgewählter Bestände in der Wienbibliothek im Rathaus nach. Einblattdrucke, Flugblätter und Flugschriften sind bedeutende Medien in der Kommunikationskultur seit Beginn der Frühen Neuzeit und können als Massenkommunikationsmittel verstanden werden, insbesondere seit der Erfindung des Buchdrucks. Die Definitionen und Begriffsfindungen, die zu Beginn dieser Arbeit ausgeführt werden, fassen die charakteristischen Merkmale und Gemeinsamkeiten dieser historischen Sondermaterialien zusammen. Den Hauptteil der Arbeit bildet die Bestandsanalyse, die ausgewählte Bestände der Wienbibliothek in Hinblick auf deren Bestandsge- 
schichte, Erschließungs- und Digitalisierungsstand genauer untersucht. Nicht nur die Erschließung, sondern auch die Verwahrung und Präsentation werden hierbei in den Fokus genommen. Eine sukzessive Aufarbeitung, Verfügbarmachung und Digitalisierung dieses Materials stellt für die Forschung eine wertvolle Ressource dar und verdeutlicht den historischen Quellenwert dieser Sonderbestände.

\section{Dipl.-Ing. ${ }^{\text {in }}$ Dr. ${ }^{\text {in }}$ Anna Soja}

\section{Informationsstrukturen an Bibliotheken zur Nutzung digitaler Ressourcen am Beispiel „Library Labs“. Empfehlungen für die Konzeption eines „Library Labs" an der Österreichischen Nationalbibliothek}

Umfangreiche Bestände an digitalen Ressourcen bringen für Bibliotheken große Herausforderungen: einerseits die langfristige Bewahrung und Aufbereitung, andererseits der Bedarf an Informationsinfrastrukturen, um bestmöglichen Zugang zu den Beständen zu ermöglichen. Library Labs dienen genau diesem Zweck: als Serviceeinrichtung für Wissenschaft, Kunst und Öffentlichkeit im Rahmen einer Bibliothek schaffen sie Zugriff auf die Sammlungen und Daten und ermöglichen neue Nutzungsmöglichkeiten. Ziel dieser Master-These war es, Vorschläge für die Schaffung eines Library Labs für digitale Geisteswissenschaften (DH) an der Österreichischen Nationalbibliothek (ÖNB) zu erarbeiten.

In der These wird der Begriff Library Lab abgegrenzt, ein Überblick über die digitalen Geisteswissenschaften geboten und auf die Beziehungen von Bibliotheken, im Speziellen der ÖNB, zu den DH eingegangen.

Um Empfehlungen für die Erstellung eines Konzepts für ein Library Lab an der ÖNB abgeben zu können, wurde das entsprechende Umfeld in Österreich analysiert. Weiters wurden Beispiele von Library Labs an anderen National- und Universitätsbibliotheken beschrieben und durch einige Interviews von Verantwortlichen untermauert. Die Bedürfnisse der Nutzenden bezüglich Library Labs wurden mittels eines Literaturüberblicks und einer standardisierten qualitativen $\mathrm{DH}$-ExpertInnenbefragung erfasst.

Die Ergebnisse dieser Analysen ergaben zahlreiche Hinweise, was beim Aufbau eines Library Labs an der ÖNB zu berücksichtigen wäre, wenn die Nutzenden und ihre Bedürfnisse im Vordergrund stehen sollen. Diese potentiellen KundInnen zu erreichen, auch wenn sie nicht direkt an die Institution angebunden sind, sollte über breite Informationsstreuung erreicht werden. Für Marketing, Administration und wissenschaftliche Betreuung 
wäre eine Projektmanagerln vorzusehen, unterstützt von einer IT-Expertln für Schnittstellen, Tools und technische Betreuung. Die Auswahl und Aufbereitung der Inhalte sollte in Zusammenarbeit mit den Sammlungen erfolgen und nationale/internationale Kooperationen und Vernetzung dürfen nicht vernachlässigt werden.

Durch die Umsetzung der Erfahrungen verwandter Institutionen und fachkundiger NutzerInnen kann die ÖNB mit einem Library Lab ein neues Service für Forschung und interessierte Öffentlichkeit bereitstellen und sich selbst mit dieser Informationsinfrastruktur als Innovationsträger positionieren.

\section{Dr. ${ }^{\text {in }}$ Gabriele Pum Österreichische Nationalbibliothek, Ausbildungsabteilung E-Mail: gabriele.pum@onb.ac.at}

Dieses Werk ist lizenziert unter einer

Creative-Commons-Lizenz Namensnennung 4.0 International 